\title{
Limited aperture light source streak photography
}

\author{
M. S. Vassiliou and Thomas J. Ahrens \\ Seismological Laboratory 252-21, California Institute of Technology, Pasadena, California 91125 \\ (Received 18 June 1981; accepted for publication 16 September 1981)
}

In shock wave equation-of-state experiments employing inclined mirror techniques, limited aperture light sources such as the xenon flash lamp are generally more convenient to use than extended light sources employing explosives, but can create alignment problems when mirror inclination angles are large. These problems can be solved by employing a wedge-shaped inclined mirror in place of a flat one.

PACS numbers: $07.68 .+\mathrm{m}, 46.30 . \mathrm{My}, 43.40 .+\mathrm{s}$

We describe a simple technique which has facilitated equation-of-state measurements of solids using the 40$\mathrm{mm}$ propellant gun at the California Institute of Technology shock wave facility. The technique is a modification of the well-known inclined mirror technique for measuring the free surface velocity of a shock loaded solid'; in essence, the flat mirror is replaced by a wedgeshaped one. The inclined wedge alleviates the severe alignment problems encountered with a simple inclined mirror when the light source used is of limited aperture, such as is the case with the Xenon flash lamp ${ }^{2}$ used in our laboratory. The technique increases the tolerance for mirror inclination angles of the system from about $2^{\circ}$ to $12^{\circ}$ or more, thus increasing the effective aperture of the xenon light source from $4^{\circ}$ to $\simeq 24^{\circ}$.

Mirror techniques in general depend on the change in specular reflectivity of a mirror as the lapped surface of a driver plate is driven into it by the shock-induced particle velocity. This interaction is recorded on film by a high speed streak camera. Hence, the entrance of a shock into a sample may be recorded by means of arrival mirrors placed on the sample next to the driver plate. Sim- ilarly, the exit of the shock from the sample may be monitored with buffer mirrors placed on top of the sample. In both these cases, arrivals on the streak record are perpendicular, ideally, to time lines. In the case of an inclined mirror, one obtains an inclined record which monitors the motion of a point of collision of the free surface with the mirror. The angle of inclination $\gamma$ of the resulting streak with the perpendicular to the time lines is related to the angle of inclination $\alpha$ of the mirror with respect to the sample, the writing rate $W$ of the camera, and the velocity $U_{\mathrm{fs}}$ of the sample's free surface by

$$
\gamma=\arctan \left\{W \tan \alpha / m U_{\mathrm{fs}}\right\},
$$

where $m$ is the film image magnification factor for the spatial dimension (see Ahrens and Gregson ${ }^{3}$ for a description of corrections to be applied when the shock is tilted with respect to the sample).

When $\gamma$ is extremely small, measurements of $U_{\text {fs }}$ are more error prone, and the resolution of two-wave structures is more difficult. Relatively large values of $\gamma$, however, require correspondingly large values of $\alpha$ for typical combinations of $W$ and $U_{\mathrm{fs}}$. In general $\alpha$ is limited by the



FIG. 1. Experimental setup. The gun-launched projectile impacts the sample assembly. Light from the xenon flash lamp is reflected onto the sample assembly by the fixed "turning mirror," which also reflects light from mirrors in the sample assembly to the streak camera. 


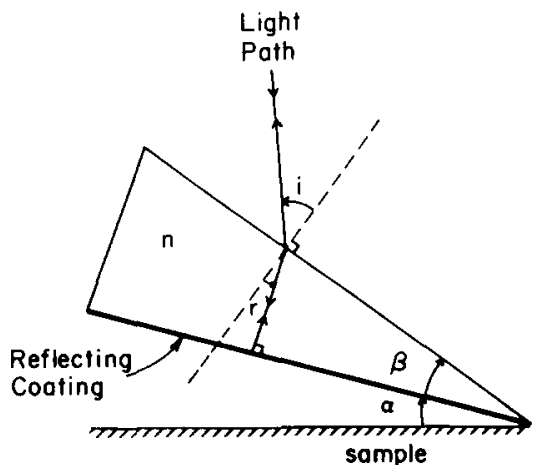

FIG. 2. Geometry of inclined wedge. Wedge is made of glass with refractive index, $n$, and has a reflecting coating on its bottom edge. $i$ $=$ angle of incidence of light from turning mirror, $r=$ angle of refraction. $\alpha=$ angle of inclination of wedge, $\beta=$ internal wedge angle; $\alpha$ and $\beta$ must be chosen such that incoming rays are reflected back along the same path with which they enter.

condition that the velocity $V_{a}$ of the point of collision must be large enough that the flow in both media is everywhere supersonic. The velocity $V_{a}$ is given by Duvall and Fowles' as

$$
V_{a}=U_{\mathrm{fs}} / \sin \alpha .
$$

Usually one requires that $V_{a}$ be greater than the maximum shock velocity $U_{s}^{m}$ in the system, which leads to a maximum allowable $\alpha$ of

$$
\alpha_{\max }=\arcsin \left(U_{\mathrm{fs}} / U_{s}^{m}\right) .
$$

Values of $\alpha_{\max }$ typically range from $15^{\circ}$ to $30^{\circ}$. For an example with $U_{\mathrm{fs}}=1.7 \mathrm{~km} / \mathrm{s}, U_{s}^{m}=6.8 \mathrm{~km} / \mathrm{s}, W=7$ $\mathrm{km} / \mathrm{s}$, and $m=0.7$ (taken from an actual experiment), $\alpha_{\max } \simeq 14^{\circ}$, with a corresponding $\gamma$ of about $56^{\circ}$.

With a simple flat inclined mirror, large values of $\alpha$ can cause alignment problems without an extended light source. The reflected rays from the inclined mirror deviate too much from those coming from the arrival and buffer mirrors. In the laboratory configuration shown in Fig. 1, values of $\alpha$ greater than $1.5^{\circ}-2^{\circ}$ cannot be tolerated. Use of an extended light source, such as an argon candle (see e.g., Keeler and Royce ${ }^{4}$ ), instead of a limited aperture xenon flash lamp, might solve this problem. Such light sources, however, are difficult to fire in synchronization with the impact of a gun-launched projectile.

A simple solution is to replace the inclined mirror by an inclined wedge. The wedge opens out toward the fixed "turning mirror" (Fig. 1), which reflects light from the xenon lamp onto the target assembly. The wedge can be constructed such that some rays hitting it can be reflected back to the turning mirror approximately parallel to their original paths, and then reflected by the turning mirror

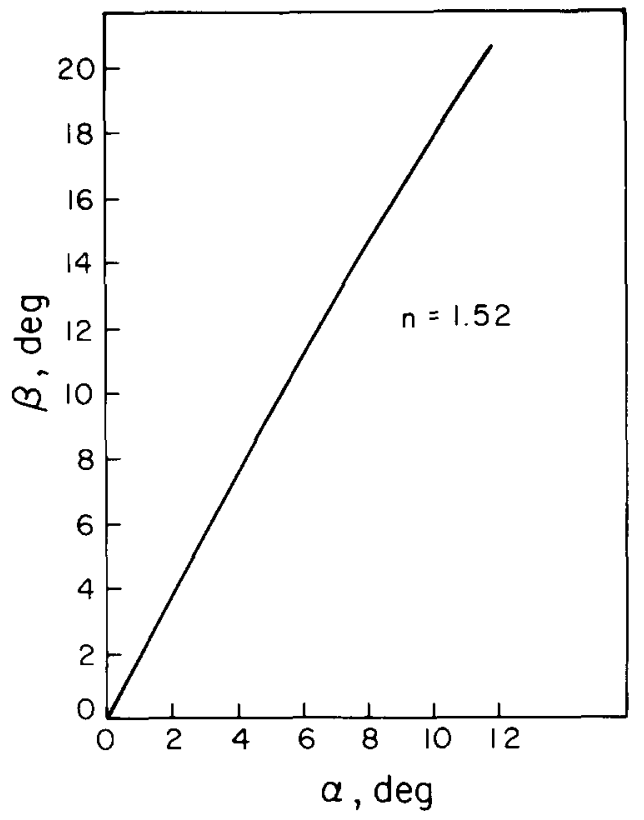

FIG. 3. Required internal wedge angle $\beta$ for a given inclination angle $\alpha$, for a wedge made of glass with refractive index $n=1.52$.

to the camera port, which also receives the rays from the buffer and arrival mirrors.

The simple geometry of the inclined wedge is shown in Fig. 2. For a ray to be reflected in a path exactly parallel to its path of entry, it must be refracted by the wedge's upper surface such that it strikes the lower reflecting surface normally. For this condition to be satisfied, the angles of incidence and refraction at the upper surface can, respectively, be $(\alpha+\beta)$ and $\beta$. Snell's law then gives

$$
n=\sin (\alpha+\beta) / \sin \beta \text {. }
$$

An appropriate wedge angle corresponding to a desired inclination angle can thus be very easily obtained. A sample curve of $\beta$ vs $\alpha$ for a glass wedge with $n=1.52$ is shown in Fig. 3.

This work was supported by the Earth Sciences Section, National Science Foundation, Grant \#EAR 7906766. (Contribution \#3595, Division of Geological and Planetary Sciences, California Institute of Technology).

' G. E. Duvall and G. R. Fowles, "Shock Waves," in High Pressure Physics and Chemistry, Vol. 2, edited by R. J. Bradley

(Academic, New York, 1963).

${ }^{2}$ T. Goto, G. R. Rossman, and T. J. Ahrens, "Absorption Spectroscopy in Solids Under Shock Compression," in High Pressure Science and Technology, Vol. 2, edited by K. D. Timmerhaus and M. S. Barber (Plenum, New York, 1979).

${ }^{3}$ T. J. Ahrens and V. G. Gregson, Jr., J. Geophys. Res. 69, 4389 (1964).

${ }^{4}$ R. N. Keeler and E. B. Royce, "Shock Waves in Condensed Media," in Physics of High Energy Density, edited by P. Caldirola and M. Knoepfel (Academic, New York, 1971). 